\title{
Tuiles détachées de Jean-Christophe Bailly : métaphores de l'autoportrait
}

\section{Brigitte Ferrato-Combe}

\section{(2) OpenEdition}

12 Journals

Édition électronique

URL : http://journals.openedition.org/recherchestravaux/376

DOI : 10.4000/recherchestravaux.376

ISSN : 1969-6434

Éditeur

UGA Éditions/Université Grenoble Alpes

\section{Édition imprimée}

Date de publication : 30 décembre 2009

Pagination : 67-79

ISBN : 978-2-84310-159-5

ISSN : 0151-1874

\section{Référence électronique}

Brigitte Ferrato-Combe, "Tuiles détachées de Jean-Christophe Bailly: métaphores de l'autoportrait », Recherches \& Travaux [En ligne], 75 | 2009, mis en ligne le 30 juin 2011, consulté le 08 septembre 2020 URL : http://journals.openedition.org/recherchestravaux/376 ; DOI : https://doi.org/10.4000/ recherchestravaux.376 


\section{Tuiles détachées de Jean-Christophe Bailly : métaphores de l'autoportrait}

L'ouvrage de Jean-Christophe Bailly, Tuiles détachées, inaugure la collection "Traits et portraits» en février 2004. Il le fait en proposant une réflexion fine et subtile sur "l'exercice de l'autoportrait», réflexion qui se déploie à travers un réseau extrêmement dense de métaphores. Ces métaphores tentent de décrire l'entreprise dans laquelle l'auteur s'est lancé, et qui est définie en quatrième de couverture comme un «autoportrait vagabond».

S'agit-il pour autant d'un autoportrait fragmentaire? À première vue, le choix de cet ouvrage peut surprendre, car il se présente sous la forme d'un texte continu de cent vingt pages compactes, ponctué seulement d'une dizaine d'illustrations. Ces photographies ou dessins, en noir et blanc, sont pour la plupart sagement disposés sur des pages distinctes, alors que d'autres volumes de la collection, comme Qui est là de Christian Lacroix, ou $\grave{A}$ la couleur de Jan Voss jouent à fond le jeu de la discontinuité et du collage de fragments.

La lecture du texte, cependant, amène à nuancer cette première impression, et plus encore une étude un peu attentive des métaphores qu'il recèle. Comme le suggère Paul Ricour à propos de toute "métaphore vive ${ }^{\mathrm{I}}$ ", celles que déploie Jean-Christophe Bailly proposent en effet une re-description de l'objet qu'elles désignent et amènent à le considérer d'un œil neuf. Cet objet modelé par le travail métaphorique dans Tuiles détachées est triple : c'est tout à la fois le sujet qui s'analyse et se souvient, la mémoire scrutée dans son

I. P. Ricour, La Métaphore vive, Seuil, «L'Ordre philosophique», 1975, en particulier la $7^{\mathrm{e}}$ étude : "Métaphore et référence». 
fonctionnement, et enfin le texte dans et par lequel s'élabore l'autoportrait. Si certains réseaux métaphoriques imposent l'image d'un flux continu, en accord avec la présentation matérielle du texte, ils coexistent avec d'autres, plus nombreux, qui suggèrent au contraire la fragmentation, la diversité, une structure lacunaire, un parcours aléatoire et contradictoire. Il s'agit ici d'analyser comment les nombreuses métaphores à travers lesquelles JeanChristophe Bailly donne à voir son autoportrait sont régies, aussi bien sur le plan sémantique que sur celui de leur organisation syntaxique et textuelle, par cette double aspiration au continu et au fragmentaire.

Une photographie insérée dans le texte ${ }^{2}$ propose une assez bonne image de cette tension entre continuité du flux et dispersion des fragments : il s'agit d'un fragment d'écorce de bouleau dont la surface unie est ponctuée de traits inégaux en longueur et en épaisseur, répartis de façon aléatoire, qui cependant semblent tous emportés par un même mouvement. La plupart des métaphores sont filées d'un bout à l'autre de l'ouvrage, sans l'être pour autant de façon continue ni exclusive. Le "fil rouge» mentionné dès les premières lignes disparaît assez vite, image trop unificatrice et sans doute aussi trop figée. Celle des fils qui s'entrecroisent et s'emmêlent la remplace, image moins linéaire et plus pertinente, qui impose très tôt la représentation d'un sujet complexe et précaire :

Là où nous cherchions l'image d'un sujet [...] là où nous avions besoin d'un contour, nous sentons que nous ne sommes faits que d'une infinité de franges qui s'enchevêtrent et se dérobent. (p. Io)

Le sujet s'effiloche tandis que la vie, dans sa profusion, apparaît comme une "sorte de tissu très serré composé d'une infinité de fils» (p. 70). Solidité et continuité de l'ensemble tissé, fragilité de "chaque fil individuel» qui «se chine continûment avec quantité d'autres semblables à lui dans leur principe, jusqu'à ce qu'enfin il casse» : la métaphore du tissu - qui décrit ici, explicitement, la vie mais suggère, inévitablement, le texte - réaffirme la tension entre continuité et discontinuité, comme l'image de l'écorce de bouleau qui la précède de quelques pages et pourrait en être une figuration.

Ce «tissu très serré» de la vie - ou du texte - est perçu d'abord comme un "ruissellement infini» (p. 70) : la fluidité qui le caractérise l'inscrit dans le réseau métaphorique de l'eau, qui domine très largement dans Tuiles détachées. C'est en effet à une image aquatique que Jean-Christophe Bailly recourt en premier lieu pour définir son cogito personnel, la conviction intime de sa

2. J.-Chr. Bailly, Tuiles détachées, Mercure de France, "Traits et portraits», 2004, p. 66 : photographie d'une écorce de bouleau. Les références ultérieures à cet ouvrage seront indiquées entre parenthèses après chaque citation. 
propre existence qui naît de la conscience de la pensée. Relevant moins du rationalisme cartésien que de la "pensée rêveuse ${ }^{3}$ " définie par J.-B. Pontalis, cette pensée trouve son origine dans l'enfance, et non dans l'expérience adulte de la réflexion :

Le cogito, mais comme une pensée d'enfance, une bouée où l'on s'accroche, quelque chose d'à peine plus dense que la conscience de respirer, quelque chose qui respire et qui dit tout bas que l'on est, que l'on pense, que l'on y pense, que l'on est ce doute qui pense ou se penche, allongé, sur lui-même [...]. (p. 8)

L'image de la bouée, que l'on pourrait d'abord lire comme une image figée, ou motivée seulement par la référence à l'enfance, prend pleinement son sens lorsque, quelques lignes plus loin, l'expérience du cogito est décrite successivement comme "une mare où la pierre du sujet («moi») a été jetée, mais pour aussitôt faire des ondes », « un forage», «un étang d'où, pour finir, lentement puis plus vite, s'échapperaient des bulles». Plus encore lorsque la petite mare sur la plage de Beg-Meil, souvenir privilégié et enchanté des vacances enfantines, devient métaphore de l'écriture autobiographique :

$\mathrm{Au}$ fond, ce que je fais ici, ce n'est rien d'autre que ce que je faisais enfant en lançant de petits bateaux [...] sur une mare d'eau de mer comprise entre des rochers, en Bretagne. Seul compte ici le sillage, et que ce qui revient s'en retourne à la citerne vivre sa vie de souvenir [...]. (p. 33)

Cette mare, pour reprendre les analyses bachelardiennes ${ }^{4}$, est à la fois eau morte et eau vive : eau stagnante de la «mare où la pierre du sujet («moi») a été jetée» (p. 8), eau sombre et mystérieuse de la «citerne» - images de la mémoire et même de l'inconscient ${ }^{5}$ - mais aussi eau vive, animée d'un mouvement qui est à la fois celui du souvenir et celui de l'écriture : les «ondes» provoquées par la pierre, le «sillage» du bateau lancé par l'enfant, le mouvement du «nageur» autobiographe :

Le mouvement est donc celui de l'autobiographie qui, avant d'être un genre, est un mode - celui du sujet qui se souvient, celui du nageur qui tantôt fait la planche et tantôt plonge dans le flux de ses souvenirs. (p. II)

3. Le sujet emblématique de cette "pensée rêveuse» pourrait être ce "dormeur éveillé ", emprunté au Songe de Constantin de Piero della Francesca, qui donne son titre au volume de la collection «Traits et portraits» publié par J.-B. Pontalis quelques mois seulement après celui de J.-Chr. Bailly.

4. G. Bachelard, L'Eau et les rêves. Essai sur l'imagination de la matière, José Corti, 1942.

5. On trouvait déjà une image semblable dans les dernières pages de la Description d'Olonne, où est évoquée la "gaffe du souvenir " : «[...] des hommes sont au-dessus d'un marais, sur des barges, ils cherchent quelque chose sous l'eau en s'aidant de longues perches de bois [...]». (J.-Chr. Bailly, Description d'Olonne, Christian Bourgois, I992, p. I90). 
Le sujet n'est plus ici la "pierre» jetée dans l'eau d'une mare, sujet passif de l'expérience du cogito, mais un "nageur" qui évolue librement entre surface et profondeur, alternant un mouvement volontaire - la plongée - et une attitude délibérément passive de réceptivité pure - la planche.

C'est ensuite une image située au confluent lexical et imaginaire du tissu et du flot que propose le texte de Jean-Christophe Bailly, celle des "nappes»: «........ voilà, oui, ce sont des nappes, et je les tire vers moi, il n'y a pas à se forcer beaucoup, elles viennent d'elles-mêmes [...].» (p. 24-26). Si la nappe que l'on tire vers soi est plutôt en tissu (le mot est d'ailleurs apparu pour la première fois dans le contexte de l'atelier de la tante couturière où l'enfant était fasciné par "le glissement des tissus» et "comme par nappes [...] happait les mots et les massifs de mots du monde divisé des adultes» [p. 24], la photographie qui accompagne les premières occurrences de ce mot entraîne vers une lecture différente, et impose l'image d'une étendue d'eau (même si, devant cette photographie des chutes d'Iguazu, c'est le mot «rideau» plutôt que celui de "nappe» qui pourrait venir à l'esprit $\left.{ }^{6}\right)$. Cette photographie, dont l'emplacement n'est certes pas dû au hasard, joue un rôle déterminant dans l'élaboration du sens du texte au moment de la lecture, comme elle l'a sans doute joué au moment de l'écriture. C'est l'isotopie du fluide qui domine à nouveau et qui unifie un ensemble complexe de métaphores par lesquelles se disent à la fois l'émergence des souvenirs et leur verbalisation, passant sans solution de continuité du registre aquatique à celui de la syntaxe ou du cinéma :

[...] les souvenirs viennent en masses fractionnées, c'est la phrase qui tourne avec eux : il y a une hélice, un mouvement, un sillage, mais nous sommes en pleine mécanique des fluides, c'est comme une sorte de ralenti discontinu ou comme un fondu enchaîné parfois flou, un paysage de turbulences et de surimpressions ou encore, et plus exactement si l'on doit garder la métaphore du cinéma, ce sont des rushes qui défilent, sans montage, mais comme portés par des courants. (p. 26)

Le mouvement de l'eau, le flux verbal de la phrase, le flux d'images des rushes cinématographiques sont autant de figurations d'une sorte de flux mental qui constitue le sujet au moment où il prend conscience de sa propre existence. C'est en ces termes que Jean-Christophe Bailly reformule son cogito personnel. Récusant le dispositif optique de Descartes, dont il insère une image dans les premières pages de son livre ${ }^{7}$, il propose sa propre version de l'accès à la conscience de soi :

6. Vue partielle des chutes d'Iguazu en Argentine, photo de l'auteur, p. 25 et p. IOI (détail).

7. Descartes, Les Météores, reproduction p. II. 
Dans la scène optique qui le confirma [le cogito cartésien], le sujet existait comme un point. Un homme tient un bâton, regarde un arc-en-ciel, plonge son bâton dans l'eau. [...] Mais il s'agit là d'un monde de l'idée, d'un pur dessin de corps, de lignes et d'angles. Or ce n'est pas ainsi que nous vivons, nous ne tombons pas dans le monde comme un pur regard, mais comme une pensée, ou comme une phrase [...] Pensée sans langage au commencement, avant l'apprentissage, mais dans le silence et les balbutiements de laquelle s'initie la phrase, d'emblée infinie, qui va nous accompagner jusqu'à la fin. (p. Io)

Se projeter dans le monde comme une phrase et non comme un point : c'est dire que la dimension syntaxique, au sens - étymologique - du lié, est constitutive d'un sujet, dont se formule par ailleurs, comme on le verra plus loin, la fragmentation. Cette association du continu et du discontinu s'appréhende aussi bien dans le registre de la syntaxe que dans celui du cinéma : le "film verbal» (p. Io) ou la "phrase-film» (p. iI) dont parle Jean-Christophe Bailly sont à la fois la pellicule qui se déroule sans interruption et les images juxtaposées qui viennent s'y enregistrer. Le sujet est défini à plusieurs reprises comme une «surface d'inscription» (p. I2), "espace d'un dépôt continu» (p. I2), surface sensible comparée tantôt à un ruban de machine à écrire tantôt à une pellicule photographique, les deux images fusionnant dans cet éloge de «la marche sans but", de "la promenade" souvent célébrée par Jean-Christophe Bailly comme son activité favorite, très intimement liée à l'écriture :

Parfois même, la promenade, si c'est le mot, atteint à une force traversante, on a l'impression qu'entre le ruban intérieur et ce qui vient s'imprimer sur lui plus rien ne s'interpose et ne fait obstacle : même si à ces moments-là, on n'écrit pas, on est tout entier dans la frappe où le ruban sursaute et peut-être bien y a-t-il, au fond, pour l'écriture, un temps en retrait qui serait celui de l'impression et un temps actif qui serait celui du développement. (p. II6)

Que le cogito ne soit pas défini comme un dispositif optique ne diminue en rien l'importance du regard pour Jean-Christophe Bailly. Dans Tuiles détachées, il évoque l'importance qu’ont eue pour lui les images à collectionner, comme ce "Palmier traversé par une planche, figure de l'album NestléKohler Les merveilles du monde", reproduit page 53. Ces vignettes, trouvées dans les plaquettes de chocolat et collées dans des albums, constituent les premiers fragments d'une culture encyclopédique, complétées par les "panneaux muets " des «leçons de choses» et par un abécédaire associant à chaque lettre une image. La découverte du monde par l'image accompagne ainsi la découverte du langage puis de l'écriture, sur le mode du fragmentaire et du discontinu, du «feuilletage infini» (p. 56). Jean-Christophe Bailly retrouve ici la tradition de l'autoportrait comme genre encyclopédique, speculum mundi, telle que l'analyse Michel Beaujour ${ }^{8}$ : le sujet se constitue de ce qu'il

8. M. Beaujour, Miroirs d'encre. Rhétorique de l'autoportrait, Seuil, «Poétique», 1980, p. 29-4I. 
a vu, lu, observé, compris, et son portrait reflète le monde. Les reproductions d'œuvres d'art dans la revue L'CEil, comme ce portrait de Miró au musée de la Marine de Barcelone photographié par Brassaï (p. 57), viennent ensuite élargir le champ des possibles, ouvrir à l'expérience artistique. JeanChristophe Bailly est un contemplateur passionné de la peinture, comme en témoigne, parmi d'autres, L'Atelier infini ${ }^{9}$, ouvrage qui en reprend et complète un autre au titre encore plus évocateur : Regarder la peinture ${ }^{\mathrm{IO}}$. Avant de décrire minutieusement les tableaux qu'il a choisis dans une histoire de l'art dont il repousse au maximum les limites temporelles et géographiques, Jean-Christophe Bailly y analyse longuement la question primordiale pour lui, du regard porté sur "les choses» directement ou par l'intermédiaire de leur représentation. Et c'est en détournant une parole de peintre, la célèbre formule de Poussin «moi qui fais profession de choses muettes", qu'il définit l'écrivain comme "le porte-voix de l'insistance muette des choses" (Tuiles détachées, $\mathrm{p} . \mathrm{IO}^{\mathrm{II}}$ ).

Mais ce regard, à la différence peut-être de celui du sujet cartésien, n'est pas celui d'un observateur statique. La dernière image du livre est une photographie de Jean-Christophe Bailly vu de dos, se promenant dans Rome. Cet "homme de $\operatorname{dos}^{12}$ », comme l'a montré Georges Banu, nous apparaît dans la position du spectateur et c'est bien un autoportrait en spectateur, en contemplateur du monde, que Jean-Christophe Bailly nous livre avec Tuiles détachées. Il enracine sa vocation d'écrivain dans l'observation du réel et dans le compte rendu qu'il en faisait, enfant puis jeune homme, à l'intention de son père devenu aveugle, ayant conclu avec celui-ci un "contrat [par lequel il devait], par le langage, suppléer à sa vue absente» :

Ce contrat avec mon père, $j y$ vois une origine possible de ce que je suis devenu : en effet, j'eus très tôt la sensation que je devais regarder le monde pour lui, qui ne le pouvait plus. (p. 93)

Pour bien regarder le monde, il faut l'arpenter : le fils prête à son père non seulement ses yeux, notamment pour la lecture, mais aussi son corps libre de déambuler au gré de ce qu'il voit; il se fait promeneur et part à la découverte de l'espace pour permettre à son père de le redécouvrir mentalement :

Mais l'essentiel de cette activité passait par des promenades que je faisais à Paris et que je racontais ensuite à mon père, qui s'efforçait de son côté d'en reconstituer les parcours par le souvenir [...]. (p. 93)

9. J.-Chr. Bailly, L'Atelier infini, Hazan, 2007.

Io. Id., Regarder la peinture, Hazan, 1998.

II. On pense aussi au titre de son essai sur les portraits du Fayoum : L'Apostrophe muette, Hazan, 2005.

I2. G. Banu, L'Homme de dos, Adam Biro, 2000. 
Convertir choses vues et itinéraires en descriptions et en récits, c'est l'occasion de prendre conscience du pouvoir du langage et de la séduction de l'écriture :

[...] je découvrais chez moi le plaisir que j’avais ainsi à lire et à raconter, à décrire. [...] je puis dire que c'est en partie auprès de mon père, qui rêvait pour moi d'une vie active, que se forma l'espace où je décidai de devenir écrivain. (p. 93-94)

C'est peut-être au cours de ces promenades dans la ville, et de la mise en récit de ces itinéraires, qu'a germé le projet de la Description d'Olonne, paru en 1992. Cet ouvrage singulier est consacré à la description minutieuse du dédale des rues et des bâtiments d'une ville qui ne figure sur aucune carte mais se met à exister avec force dans l'esprit du lecteur au fil des pages. Le narrateur, censé y avoir passé trois années de sa vie, se livre à une déambulation au gré des souvenirs, inaugurant un genre nouveau de récit, la "promenade rétrospective» caractérisé par "la précision topographique et une relative retenue dans les épanchements ${ }^{13}$ ". Un détour par Olonne s'impose pour bien saisir les enjeux de l'autoportrait de 2004. Jean-Christophe Bailly lui-même l'accomplit puisqu'il reproduit dans Tuiles détachées le plan $\mathrm{d}^{\prime} O$ Olonne ${ }^{\mathrm{I} 4}$ et se livre à quelques analyses sur cet ouvrage "aux frontières du roman", "sorte de maquette faite de mots et qui établissait la fiction sur un mode absolu» (p. 82). La Description d'Olonne apparaît à la fois comme une dérobade devant le récit autobiographique, voire un refus du récit dans son principe, et comme la préfiguration de ce que peut être un autoportrait fragmentaire et indirect. La forme d'une ville - qui doit beaucoup à Gracq et à Nantes ${ }^{15}$ - se substitue à la forme d'une vie ${ }^{16}$, donnant à voir par les méandres de ses rues, la diversité de ses quartiers, la gaieté ou la mélancolie de son atmosphère, tout un paysage intérieur. La description se substitue au récit autobiographique, pour révéler une intimité sans passer par la confidence et l'anecdote. L'organisation par courts chapitres thématiques, centrés sur une rue, un quartier, un monument ou un personnage célèbre de la ville, exclut toute structure chronologique et tout récit continu :

13. Description d'Olonne, op. cit., p. 8.

I4. Illustration p. 82. Ce plan était fourni sur une feuille séparée avec la Description d'Olonne, qu'il a suscitée. Il fait partie d'une série de dessins labyrinthiques de villes imaginaires, réalisés au crayon puis au feutre, d'une manière un peu automatique que J.-Chr. Bailly apparente aux "dessins de téléphone" (Tuiles détachées, p. 8I).

15. J. Gracq, La Forme d'une ville, José Corti, 1989. On peut lire dans Tuiles détachées, p. 82 : "Cette ville décidée le long d'un estuaire avait une forte déclinaison atlantique, c'était un peu comme si entre Nantes et Bordeaux il y avait eu un autre fleuve et qu'il ait généré lui aussi une grande cité.»

I6. " $\mathrm{Si}$ je ne suis pas revenu à Olonne, c'est parce que je sais que la forme de ma vie y a correspondu à celle de la ville, qu'elles se sont entendues l'une et l'autre, presque jusqu'à la fin, dans une précision d'emboîtage que je ne pourrais pas retrouver" (Description d'Olonne, op. cit., p. 9). 
Bien qu'il soit sans doute impossible de ne pas faire allusion à des traits personnels - le rapport d'un individu à une ville est avant tout et d'emblée un rapport intime -, je me suis efforcé de conserver autant que possible le mode, choisi initialement, de la description. Je n'y suis parvenu qu'en partie. En procédant par approches successives, il me semblait que je pouvais du moins trouver un équilibre entre le caractère nécessairement autobiographique d'un livre de souvenirs et les motifs plus libres et plus aériens d'une sorte de monographie ${ }^{17}$.

La Description d'Olonne témoigne ainsi par avance de la réticence de JeanChristophe Bailly à l'égard du récit, et tout particulièrement du récit autobiographique, qui s'efforce de «faire venir l'eau de la citerne, une eau qui, de toute façon, suinte la nuit dans nos rêves et $s^{\prime}$ infiltre le jour dans nos pensées" (p. 27-28). Ce dispositif destiné à capter et à canaliser les souvenirs est immédiatement dénoncé comme réducteur :

Le récit est la forme-tuyau par excellence, et nous y sommes d'autant plus accoutumés que la littérature, dans l'ensemble, malgré ses efforts pour rejoindre la masse ou faire résonner la citerne, se contente le plus souvent de créer des variations de récit : elle invente des linéarités là où il n'y avait rien de tel, elle tranche dans le jeu des connexions pour n'en retenir que quelques-unes. (p. 28)

On entend là, et particulièrement dans cette formule de "forme-tuyau» empruntée à Alberto Giacometti (p. 28), un écho de la méfiance à l'égard du récit et de sa linéarité qu'a entretenue toute une génération d'écrivains, celle du Nouveau Roman, antérieure à Jean-Christophe Bailly (né en 1949) mais dont il est indéniablement l'héritier.

Cette suspicion s'exprime explicitement à plusieurs reprises. Ainsi, après quelques pages où il retrace la rencontre de ses parents, quelques épisodes de son enfance partagée en plusieurs "côtés» à la manière proustienne, il commente :

Oui, les choses, de ce côté, peuvent se raconter ainsi, mais à nouveau je vois pointer la menace de la forme-tuyau et tout ce qu'elle élimine : des régimes de sensations, des noms de lieux ou de personnes, des enchevêtrements [...]. (p. 5I)

Ailleurs, il évoque son «incapacité à écrire des romans" et «une sorte de déficit fictionnel ", ou encore, recourant à nouveau à la métaphore cinématographique, il affirme préférer le "repérage» au "scénario" (p. 70). La forme narrative est toujours perçue comme réductrice, frustrante, incapable de rendre compte de la multiplicité et de la diversité des expériences, de la "profusion» de la vie. Plus loin, après avoir fait le récit d'un épisode marquant de sa jeunesse, "sorte de col entre deux versants de [sa] vie", et conscient que les pages qui précèdent peuvent s'apparenter à "une sorte d'autobiographie intellectuelle», il exprime à nouveau ses réticences :

17. Description d'Olonne, op. cit., p. 7-8. On pense inévitablement au Perec de La Vie mode d'emploi, la ville et ses quartiers remplaçant ici l'immeuble et ses appartements : la même tension entre description et micro-récits structure la Description d'Olonne. 
Mais là encore, face à l'évocation de ces moments, je dois bien voir tout ce qu'elle a de réducteur, tout ce qui en elle cède à la tentation de la forme-tuyau. Certes, j'essaye bien chaque fois de voir s'ouvrir l'éventail tout entier, mais ce n'est que pour suivre aussitôt une seule de ses branches [...]. (p. I04)

Il faut donc renoncer - difficilement - à la totalité pour accepter de ne saisir que des «fragments de réalité» (p. 42), «un tout petit fragment du millefeuille de noms et d'images» (p. I06), «des fragments plus ou moins dilués du passé» (p. I07), autant de "traits» (p. 58) - on se souvient du titre de la collection, "Traits et portraits» - autant de "copeaux» (p. 58).

La seule issue est alors dans la mobilité. Tout est mouvant, le paysage mental qui constitue le sujet ne cesse de se recomposer, "exactement comme se redessinent les bancs de sable et les courants sur la Loire» (p. 39). Plutôt que de vouloir seulement capter par un tuyau ce qui vient du fond de la «citerne», il est vital de glisser à la surface du monde et des choses. Le «fonds d'enfance sur quoi tout repose" ne gît pas au fond de la mémoire, même si Jean-Christophe Bailly joue sur les $\operatorname{mots}^{18}$, il constitue un "dépôt dispersé dans le monde». Sa rencontre fortuite, au hasard des promenades, introduit une "dilatation du temps retrouvé», mais celle-ci n'est pas perçue comme «la remontée proustienne, révélant d'un seul coup toute une matière enfouie», plutôt comme "une élongation du temps qui ferait pression sur la mémoire, celle-ci faisant à son tour pression sur le présent» (p. 60), une mise en consonance du présent et du passé. Jean-Christophe Bailly n'oppose pas une surface qui serait vaine, trompeuse, illusoire, à une profondeur où seraient ensevelies l'authenticité des souvenirs et la richesse des sensations; surface et profondeur, sensation et souvenir entrent en communication. La métaphore privilégiée n'est pas celle de la «résurgence» mais celle du «ricochet» qui parcourt la surface et fait impact sur l'eau en des points multiples. Cette image dynamique dans son essence était déjà très présente dans la Description d'Olonne ${ }^{19}$. Elle est ici employée à propos du sujet (p. 29), de la progression de la pensée (p. 37) ou encore de l'enchaînement des associations d'idées et de souvenirs qui font progresser le texte lui-même : «[...] infini est le nombre de ricochets possibles à partir de tout lieu arpenté ou même seulement aperçu.» (p. 69). Le sujet qui se souvient - ou plus généralement le sujet qui pense, qui vit - serait donc moins une pierre jetée vers le fond de la mémoire et produisant des ondes concentriques qu'une pierre lancée à la surface du monde,

I8. p. 59. C’est moi qui souligne.

19. Dans la Description d'Olonne, l'image du ricochet est souvent associée au souvenir : p. 49, I5I, I55, I73 et enfin dans les dernières lignes : «À la surface de l'eau la pierre ricoche et l'on raconte les éclaboussures, mais où est cette eau, quelle est-elle, et se peut-il qu'elle soit tout entière dans l'esprit? Et, si oui, où est l'esprit?» (p. I90-I9I). 
accomplissant "des séries de ricochets longues et fines» (p. II7), image à la fois d'énergie, de vitesse et de mouvement, qui rejoint l'«accélération» et le "gai savoir funambulesque" caractéristiques des nuits d'ivresse passées en compagnie d'un ami, Claude Givaudan (p. 37). La projection euphorique de soi oriente un autoportrait qui s'achève - avant de conclure - par ce vœu :

Ce que je m'efforce de faire, au fond, ce n'est rien d'autre que [...] de rester fidèle à cette intensité mobile - fidèle comme on peut l'être à ce qui s'est perdu. (p. II9)

Cette «intensité mobile», c'est celle d'une jeunesse placée sous le signe des romantiques allemands et, tout spécialement, du Lenz de Büchner, que JeanChristophe Bailly édite en 1974, et qu'il célèbre à sa manière en 1978 par un acte de militantisme individuel et poétique rétrospectivement perçu comme «une sorte de col entre deux versants de [sa] vie» (p. IO2) :

Le 20 janvier 1978, cela faisait deux cents ans que Lenz était parti dans la montagne. Mon idée fut de détourner la manie commémorative pour la faire servir à cet événement secret et perdu : je réalisai une affiche où l'on pouvait lire, sous la photo floue d'un homme en manteau qui courait le long d'un grillage, le début du récit de Büchner, le tout surmonté malgré tout encore d'un slogan qui disait : La pureté existe, elle se gagne, elle est perdue. Il y a 200 ans, Lenz partit dans la montagne, à Waldbach. Je collai de nuit cette affiche dans les rues de Strasbourg, ainsi que dans le village même de Waldersbach [...]. (p. IOI-IOz)

Image de marcheur, une fois encore, cet «homme en manteau qui courait le long d'un grillage", sorte d'autoportrait indirect, trouve un double écho : l'image que le narrateur d'Olonne retient de lui-même à la fin du prologue, "cette photo où je descends le cours Cervier en essayant de marcher sur mon ombre $^{20}$ ", et bien sûr la photo de Jean-Christophe Bailly placée à la fin de Tuiles détachées: l'homme en manteau ne court plus, il marche dans les rues de Rome, mais il obéit toujours à l'impérieuse nécessité du mouvement :

Toujours est-il qu'il faut au départ un grand oui, un grand allant. Sans lui, rien ne se ferait, quelque chose de physique est attaché à ce pas, une force, une force qui traverse une ombre ${ }^{2 \mathrm{I}}$. (p. II6-II7)

20. Description d'Olonne, op. cit., p. 9.

2I. La phrase soulignée dans le texte est la reprise d'une citation faite précédemment, et commentée : «des vers de Wallace Stevens extraits d'un poème intitulé An Ordinary Evening in New Haven. Ces vers sont magnifiques, il y est dit du réel : "It may be a shade that traverses a dust, a force that traverses a shade» ("Une ombre qui traverse une poussière, une force qui traverse une ombre" p. 74-75). À rapprocher de la phrase attribuée à Sam, le peintre américain de la description d'Olonne, qui s'efforce de capter les ombres entre deux plaques de verre : "There is no time for dust on shadows" («il n'y a pas de temps pour la poussière sur les ombres disait-il», op. cit., p. 63). 
Cette mobilité, d'inspiration nietzschéenne, s'accompagne d'une certaine légèreté, et rejoint la dynamique du jeu. Si les avant-gardes, dont il reconnaît avoir eu, dans sa jeunesse, la "nostalgie», sont figurées par une métaphore agressive - «Jeter violemment et en chœur des pierres dans l'eau, des pierres qui feraient beaucoup d'éclaboussures» (p. II7) -, ce sont en définitive des images légères et ludiques, euphoriques et non tragiques, qui s'imposent : «la joie de l'éclaboussure elle-même», le «doux effet de brumisation" près d'une fontaine, un "carillon de bulles d'eau que seul le poème pourrait faire sonner» (p. II7), autant de métaphores qui disent à la fois la pluralité, la multiplicité, la fragmentation, et le bonheur du jeu - du jeu avec l'eau, notamment, qui n'est plus mare stagnante ou profondeur noire de la mémoire.

De multiples métaphores du jeu proposent, dans l'ensemble du texte, une redescription du sujet et de l'autoportrait qui met l'accent plus fortement encore sur leur caractère fragmentaire. À commencer par le jeu de mah-jong, qui fournit à l'ouvrage son titre :

........ un tuilage, une glissade... et dès lors tous les "côtés" formant entre eux une sorte de puzzle inachevé et inachevable, la mort remettant toutes les pièces du jeu dans une boîte que plus personne ne peut ouvrir. Il y a du jeu entre les pièces, il faut qu'il y ait du jeu, de l'air : non un cloisonné, mais une dissémination, un opus incertum... En fait, c'est une expression du jeu de mah-jong, et celle qui désigne les pièces qui font partie de la pioche, tuiles détachées, qui conviendrait le mieux. (p. 30)

Le titre, spontanément, pouvait s'interpréter autrement, comme l'assemblage et la superposition d'éléments distincts, de petites dimensions, formant ensemble une surface continue, ce que suggère d'ailleurs au début de la citation le mot «tuilage», Jean-Christophe Bailly jouant avec la double lecture et déjouant les attentes du lecteur. L'illustration insérée dans la même page montre un jeu composé de pièces multiples, décorées, comme autant d'images, sagement disposées comme les cartes d'une réussite. Mais le commentaire présente le mah-jong comme un jeu de mouvement, où la disposition des pièces change sans cesse, de façon à la fois calculée et aléatoire, «jeu formidable qui déséquilibre sans cesse la construction par le hasard, et réciproquement" permettant au joueur de rêver à "des combinaisons prodigieuses» (p. 3I).

Un autre jeu associe, comme le mah-jong, la fragmentation et le mouvement, et organise des déplacements aléatoires - déplacements bien réels, cette fois, du corps dans l'espace; il s'agit du jeu de marelle, utilisé par JeanChristophe Bailly comme métaphore de la vie individuelle et du texte qui la retrace, imprévisible et inimitable : 
Une vie, rien qu'une vie, ce serait justement [...] une sorte de marelle qui, ajoutant les côtés aux côtés, les surfaces aux surfaces, finirait, entre figures imposées et figures libres, par former un tracé reconnaissable et unique. (p. 49)

L'œuvre elle-même est décrite plus loin comme «une sorte de marelle désordonnée» (p. 74). Caractérisée par une extrême diversité, le passage permanent d'un genre à l'autre - essai, théâtre, poésie, etc. -, elle se définit par cette mobilité - même et la multiplicité des parcours possibles, la "distraction", voire la dispersion, n'excluant ni la "cohérence» ni l'intensité.

"Voilà. On parle de tout cela et on conclut, ou croit conclure, fermement s'il se peut. Mais ça ne tient pas, ça s'en va de partout» (p. I2I). Arrivant à l'impossible conclusion de cet impossible autoportrait, Jean-Christophe Bailly multiplie les métaphores pour en dire le caractère fragmentaire et lacunaire "oreiller crevé dont les plumes s'envolent», "bassine d'eau pleine de fentes et de trous" - mais aussi pour signifier le vertige devant la profusion et l'excès : la marelle cède la place au jeu de l'oie, au labyrinthe, au dédale, à la toupie, au tourbillon ou à la spirale. Ces images se bousculent pour figurer l'interrogation ultime sur l'écriture de l'autoportrait et sur le sujet :

Le tourment de l'autoportrait, ou du régime autobiographique qu'il suppose, je sentais confusément que je devais l'accueillir et en quelque sorte lui faire bonne figure : une sollicitation a surgi qui m'a fourni l'occasion d'une tentative, et ce qui est venu est venu, une sorte de ligne en spirale qui saute les étapes et ne lance que quelques pistes centrifuges. À la fin je me dis que l'on n'est que cela, un jeu de pistes lacunaire ou, autre métaphore, un puzzle incomplet dont les pièces ne s'emboîtent pas. (p. I22)

Devant la frustration née du vagabondage à la surface du moi et du monde, le désir s'insinue des "milliers de pages que pourrait comporter une tentative autobiographique plus longue et plus tenace» (p. I22), ou d'un ouvrage encyclopédique, sur le mode - déjà pratiqué - de l'abécédaire :

[...] pratiquement à chaque pas l'on se rend compte de ce qu'on abandonne, de ce que l'on ne se donne pas le temps de creuser, de sonder. À presque tous les mots je pourrais mettre un astérisque et renvoyer à un abécédaire où il serait défini, comme j’avais tenté de le faire dans Le propre du langage où Io 4 entrées figuraient, IO4 seulement, d'allée à yole. Mais cet autre livre expansif, peut-être impossible et dont l'organisation en tout cas devrait être toute différente et sans doute très discontinue, on ne le lira pas. Celui-ci n'en est que l'ombre, peut-être le tombeau. (p. I23)

Jean-Christophe Bailly orne ensuite d'astérisques, sans toutefois les définir, des mots porteurs de souvenirs, de sensations prêtes à affleurer : Paille*, herbe*, pin*, chaleur*, saule*, bambou*, grillon*, mots à partir desquels le texte 
pourrait à nouveau ricocher, ou encore journal de bord*, esquisse*, tableau*, fragment*, mots essentiels pour une dernière métaphore, picturale cette fois, qui dit l'inaccessible unité :

[...] le rêve de l'impossible tableau, la grande fresque que chacun porte en soi mais qui comporte aussi le risque de détruire la force de persuasion entendue dans l'esquisse ou le fragment. (p. I23)

Un des textes inauguraux de la collection "Traits et portraits", dédiée à l'autoportrait, s'achève ainsi sur un soupçon porté sur le genre dans son principe même : l'autoportrait ne serait-il pas, inévitablement, un chef-d'œuvre inconnu, nécessairement fragmentaire, nécessairement inachevé, ou peutêtre illisible, dans sa totalité, par tout autre que soi? 